\title{
Le budget global avance masqué au Parlement
}

\section{Yvonne Gilli}

Dre méd., présidente de la FMH

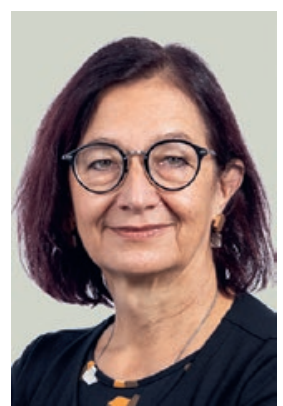

En ce moment, deux projets attirent principalement l'attention lorsqu'on parle de maîtrise des coûts dans le secteur de la santé: l'initiative pour un frein aux coûts déposée en mars 2020 par le parti du Centre et le fameux «objectif de maîtrise des coûts» présenté le 10 novembre dernier sous forme de contre-projet indirect du Conseil fédéral. Tous deux visent un plafonnement des coûts de la santé mais diffèrent beaucoup dans la manière de procéder.

L'initiative pour un frein aux coûts exige des «incitations efficaces" pour que les coûts de la santé "évoluent conformément à l'économie nationale et aux salaires moyens» [1]. Si aucune mesure contraignante n'a été arrêtée deux ans après son acceptation alors que la hausse des coûts est supérieure à $20 \%$ des salaires, «la Confédération [devrait prendre] en collaboration avec les cantons des mesures visant à faire baisser les coûts» [1]. Sans aucune précision sur les mesures exactes, l'initiative du Centre ne pouvait pas laisser le champ plus libre aux développements à venir. Dans ce contexte, la raison invoquée par le Conseil fédéral pour motiver son contre-projet peut surprendre lorsqu'il précise (pour l'initiative) que «le mécanisme proposé est trop rigide», si bien que la menace d'«un rationnement des prestations» existe "selon les modalités de mise en œuvre» [2]. C'est pourtant le contreprojet qui prévoit une régulation extrêmement stricte. Avec l'instauration d'«objectifs concernant l'augmentation maximale des coûts de l'AOS», les limites supérieures définies par le gouvernement, de manière différenciée en "groupes de coûts» et par canton, limiteraient la prise en charge médicale. En d'autres termes, les fournisseurs de prestations ne pourraient plus traiter les assurés une fois les "objectifs de coûts» atteints, sous peine de s'exposer à une intervention tarifaire de l'Etat ou à d'autres sanctions. C'est précisément ce qui fait craindre à la FMH un «rationnement des prestations médicales» [3]. Par ailleurs, l'«économie planifiée complexe et détaillée» de la Confédération ne permet pas de trouver de "réelles solutions», comme le fait remarquer le parti du Centre [4].

Malgré toutes leurs différences, ces deux projets ont en commun de vouloir imposer un budget global à notre système de santé et exigent ainsi une prise de décision fondamentale pour la politique de la santé. Or, pour l'heure, de nombreux éléments indiquent que cette discussion de fond n'aura pas lieu. Le changement de système induit par les enveloppes budgétaires prévues et les conséquences drastiques que cela implique pour la prise en charge médicale pourrait être validé dès la session d'hiver. Car, pendant que le «frein aux coûts» et les "objectifs de maîtrise des coûts» suscitent pleinement l'attention, le nouvel article 47c de la LAMal transmis par le DFI au Parlement est sur le point d'être adopté par le Conseil des Etats. Cet article prévoit lui aussi un plafonnement au sens des «objectifs de coûts» du Conseil fédéral: les partenaires tarifaires devraient fixer les coûts

\section{Alors que "frein aux coûts" et "valeur-cible»} suscitent l'attention, l'art. 47c LAMal est sur le point d'être adopté.

«justifiés» pour une année calendaire en se conformant aux exigences politiques. Si le budget global fixé à l'avance est dépassé, ils s'exposent à des sanctions financières. Le plafonnement budgétaire remettrait en question le droit à l'assurance des patients - et, en cas de doute, les médecins seraient incités à ne pas fournir de prestations pour éviter de s'exposer à des sanctions financières. A propos de l'article 47c, le Conseil fédéral précise explicitement que celui-ci vise «à ne pas dépasser cette valeur-cible» [5]. Les détails de la mise en œuvre pourraient donc être adoptés avant même que le contre-projet indirect du Conseil fédéral ne soit soumis au vote.

Reste à espérer que la Chambre haute mesure la portée de l'article 47c - et décide de l'examiner en même temps que l'initiative sur le frein aux coûts et le contre-projet du Conseil fédéral. Si ce n'est pas le cas, l'introduction d'un plafonnement des coûts pourrait être adoptée avant l'examen par le Parlement des deux grands projets dans ce sens. Une intervention d'une telle importance pour un système de santé aussi performant et apprécié mériterait une plus grande attention.

\section{Références}

La liste complète des références est disponible dans la version en ligne de l'article sur www.bullmed.ch 